Francisca Gutiérrez, Stéphanie Alenda, Nicolás Angelcos, Satisfacción y reconocimiento en ocupaciones precarias: el caso de los recolectores de basura en Chile, Izquierdas, 49, junio 2020: 848-865

\title{
Satisfacción y reconocimiento en ocupaciones precarias: el caso de los recolectores de basura en Chile
}

Satisfaction and recognition in precarious occupations: the case of waste collectors in Chile

\author{
Francisca Gutiérrez Crocco* \\ Stéphanie Alenda** \\ Nicolás Angelcos**
}

\begin{abstract}
Resumen: A partir de una encuesta y entrevistas en profundidad a recolectores de basura en Santiago de Chile, este artículo examina el sentido que otorgan estos trabajadores informales a su labor. Usando un análisis de conglomerados, el artículo muestra cinco tipos de relación de los recolectores con su trabajo. Asimismo, intenta explicar por qué la mayor parte encuentra satisfacción en esta actividad, a pesar de la precariedad que la caracteriza. El artículo plantea que esta percepción de los recolectores reposa en su aceptación de una "oferta ideológica de reconocimiento social", encarnada en la figura del "emprendimiento", la cual ha sido promovida por las políticas sociales en el país desde los años 1980. Esta aceptación estaría condicionada por los obstáculos que estos trabajadores encuentran en su trayectoria laboral, debido a su posición en el espacio social.
\end{abstract}

Palabras clave: Recolectores de basura; Recicladores; Informalidad; Reconocimiento social; Chile

\begin{abstract}
Based on a survey and in-depth interviews with waste collectors in Santiago de Chile, this paper examines the meaning that these informal workers give to their work. Using a cluster analysis, the article shows five types of relationship between collectors and their work. Furthermore, it explains why most of them find satisfaction in this activity, despite the precariousness that characterizes it. The article argues that this perception of the collectors lies in their acceptance of an "ideological offer of social recognition", embodied in the figure of "entrepreneurship", which has been promoted by the chilean social policies since the $1980 \mathrm{~s}$. This acceptance appears to be conditioned by the obstacles that these workers find in their career, due to their position in the social space.
\end{abstract}

Keywords: Waste collectors; Waste pickers; Informality; Social recognition; Chile.

Recibido: 27 enero 2019 Aceptado: 9 abril 2019

\footnotetext{
* Autora principal. Chilena. Phd en Sociología. Profesora Asistente, Facultad de Economía y Negocios, Universidad Alberto Hurtado. Investigadora Adjunta, Centro de Estudios del Conflicto y Cohesión Social (COES). fgutierrez@uahurtado.cl. La autora agradece patrocinio de FONDAP No 15130009 (COES

** Co-autora. Francesa. Directora de investigación, Facultad de Educación y Ciencias Sociales y Profesora Asociada, Carrera de Sociología, Universidad Andrés Bello.salenda@unab.cl

*** Co-autor. Chileno. Profesor Asistente, Carrera de Sociología, Universidad Andrés Bello. Investigador Adjunto, COES. nicolas.angelcos@unab.cl. El autor agradece patrocinio de FONDAP No 15130009 (COES)
} 


\section{Introducción}

La presión internacional ha aumentado en la última década para que los gobiernos latinoamericanos generen programas con el fin de reducir los residuos en las ciudades ${ }^{1}$. Existe cierto consenso en los académicos y las organizaciones no gubernamentales (ONGs) respecto a la necesidad de integrar a los recolectores informales ${ }^{2}$ en estos programas, quienes tradicionalmente han estado a cargo de esta tarea. En efecto, se estima que entre el $25 \%$ y el $50 \%$ de los residuos son reciclados en la actualidad por trabajadores informales en los países en desarrollo ${ }^{3}$. Estos hombres y mujeres recorren cotidianamente las calles de las ciudades con sus carros o triciclos, juntando los desechos antes que sean recogidos por los camiones de basura, para consumo personal y venta a empresas de reciclaje ${ }^{4}$. Alrededor del $2 \%$ de la población se dedica a esta actividad en América Latina y Asia ${ }^{5}$. La integración de estos trabajadores en el sistema formal de reciclaje tendría beneficios no solo para ellos sino también para el sistema en general, pues permitiría entregar un servicio a sectores vulnerables donde las empresas no llegan, entre otros beneficios ${ }^{6}$.

Pese a su importancia en este debate, existe un déficit de información sobre los recolectores de basura. La literatura existente se focaliza en su contribución al proceso de reciclaje y valorización del material recuperado ${ }^{7}$; su relación con el sector formal ${ }^{8}$; su proceso de organización ${ }^{9}$; la segregación social de la

${ }^{1} \mathrm{El}$ aumento de las tasas de reciclaje es uno de los Objetivos de Desarrollo del Milenio.

${ }^{2}$ Los recolectores informales reciben distintos nombres según la región y el material que recolectan. En las áreas de lengua inglesa, son conocidos como "scavengers", "waste-pickers" o "rag pickers". En Chile, reciben el nombre genérico de "cachureros" o "recolectores de basura".

${ }^{3}$ David C. Wilson, Adebisi O. Araba, Kaine Chinwah, y Christopher R.Cheeseman, "Building Recycling Rates through the Informal Sector," Waste Management (New York, N.Y.) 29, no. 2 (2009): 629-35, https://doi.org/10.1016/j.wasman.2008.06.016.

${ }^{4}$ Individuos o empresas que hacen el vínculo entre los recicladores informales y las empresas encargadas de procesar y distribuir el material reciclado.

${ }^{5}$ Wilson et al. (2009), op. cit.

${ }^{6}$ Muhammad Asim, Syeda Adila Batool, y Muhammad Nawaz Chaudhry, "Scavengers and their role in the recycling of waste in Southwestern Lahore”, Resources, Conservation and Recycling 58 (2012): 152-62, https://doi.org/10.1016/j.resconrec.2011.10.013; Thaddeus Chidi Nzeadibe y Chukwuedozie K. Ajaero, "Development Impact of Advocacy Initiatives in Solid Waste Management in Nigeria", Environment, Development and Sustainability 13, no 1 (1 de febrero de 2011): 163-77, https://doi.org/10.1007/s10668-010-9254-8; Wilson et al. (2009), op.cit; David C. Wilson, Costas Velis, y Chris Cheeseman, "Role of informal sector recycling in waste management in developing countries", Habitat International, Solid Waste Management as if People Matter Solid Waste Management as if People Matter, 30, no 4 (diciembre de 2006): 797-808, https://doi.org/10.1016/j.habitatint.2005.09.005; Peter J. M. Nas y Rivke Jaffe, "Informal Waste Management”, Environment, Development and Sustainability 6, no 3 (2004): 337-53, https://doi.org/10.1023/B:ENVI.0000029912.41481.a5; Martin Medina, "Scavenger cooperatives in Asia and Latin America", Resources, Conservation and Recycling 31, no 1 (2000): 51-69, https://doi.org/10.1016/S0921-3449(00)00071-9; Martin Medina, "Informal Recycling and Collection of Solid Wastes in Developing Countries: Issues and Opportunities”, UNU/LAS Working Paper 24 (1997).

${ }^{7}$ Maria Besiou, Patroklos Georgiadis, y Luk N. Van Wassenhove, "Official recycling and scavengers: Symbiotic or conflicting?", European Journal of Operational Research 218, no 2 (2012): 563-76, https://doi.org/10.1016/j.ejor.2011.11.030; Wilson et al. (2006), op. cit; Wilson et al. (2009), op. cit.

8 Mesharch W. Katusiimeh, Kees Burger, y Arthur P.J. Mol, "Informal waste collection and its coexistence with the formal waste sector: the case of Kampala", Habitat International 38 (2013): 1-9, https://doi.org/10.1016/j.habitatint.2012.09.002; Medina (2000), op.cit.

9 Ana Magni y Wanda Günter, "Cooperatives of waste pickers as an alternative to social exclusion and its relationship with the homeless population”, Sa Áode e Sociedade 23, no 1 (2014): 99-109; Sebastián Carenzo y María Inés Fernández Álvarez, "El asociativismo como ejercicio de gubernamentalidad: 'cartoneros/as' en la metrópolis de Buenos Aires", Argumentos 24, no 65 (2011): 171-93; Sebastián Carenzo y Pablo Míguez, "De la atomización al asociativismo: Reflexiones en torno a los sentidos de la autogestión en experiencias asociativas desarrolladas por cartoneros", Maguaré, no 24 (2010): 233-63; Maria Scarlet Do Carmo y Jose Antonio Puppim de Oliveira, "The Semantics of Garbage and the organization of the recyclers: Implementation challenges for establishing recycling cooperatives in the city of Rio de Janeiro, Brazil”, Resources, Conservation and Recycling 54, no 12 (2010): 1261-68, https://doi.org/10.1016/j.resconrec.2010.04.005; Medina (2000), op.cit; Medina (1997), op. cit. 
que son víctimas ${ }^{10}$; o algunos aspectos aislados de sus condiciones de trabajo como la precariedad de los ingresos, la desprotección y los riesgos de salud ${ }^{11}$. Sin embargo, son pocos los estudios que examinan en profundidad cómo estos recolectores informales otorgan sentido a su trabajo. Comprender la relación subjetiva de estos trabajadores con su actividad es clave para el éxito de las políticas de gestión de desechos. Una integración efectiva de los trabajadores informales al nuevo sistema depende de la capacidad que tenga éste de ofrecerles un mejor empleo, lo que implica garantizarles mejores condiciones materiales, pero también oportunidades de realización personal.

Este artículo se propone contribuir a esta discusión, a partir del estudio del caso chileno. En base a una encuesta realizada en Santiago durante los años 2013 y 2014, examinamos cómo los recolectores perciben su trabajo y las diferencias que pueden generarse en este plano al interior de este grupo. Para profundizar en esta experiencia, conducimos entrevistas. En específico, examinamos los mecanismos que permiten a estos recolectores sentirse satisfechos con su trabajo a pesar de la desprotección y estigmatización que pesa sobre esta actividad.

El artículo se divide en cuatro partes: en la primera, desarrollamos las bases teóricas que permiten analizar el sentido que otorgan estos trabajadores a su labor; en la segunda, describimos el contexto en el cual se inserta este estudio, en la tercera, la metodología utilizada; por último, presentamos los resultados.

\section{El sentido otorgado a las ocupaciones informales}

El trabajo de los recolectores informales de desechos en el mundo se desarrolla normalmente en condiciones de extrema precariedad. Están sometidos a altos riesgos sanitarios, perciben escasos ingresos, no gozan de protección ni seguridad laboral y suelen ser estigmatizados por la sociedad ${ }^{12}$. Además, estos trabajadores suelen pertenecer a las capas sociales más perjudicadas. Se trata de una actividad prioritariamente de inmigrantes y personas con bajo nivel escolar que, por estas mismas razones, difícilmente pueden insertarse en el sistema formal ${ }^{13}$.

Investigaciones recientes sobre la informalidad han cuestionado la parcialidad con que muchos estudios abordan este tipo de actividades. Denunciando exclusivamente las condiciones materiales del trabajo

\footnotetext{
${ }^{10}$ Natalia Cosacov y Mariano D. Perelman, "Struggles over the Use of Public Space: Exploring Moralities and Narratives of Inequality. Cartoneros and Vecinos in Buenos Aires", Journal of Latin American Studies FirstView (2015): 1-22, https://doi.org/10.1017/S0022216X15000425.

${ }^{11}$ Katusiimeh et al., op. cit; Asim et al. op.cit; Carenzo y Álvarez, "El asociativismo como ejercicio de gubernamentalidad”; Do Carmo et al. op. cit; Carrie L. Mitchell, "Altered landscapes, altered livelihoods: The shifting experience of informal waste collecting during Hanoi's urban transition", Geoforum, Placing Splintering Urbanism, 39, no 6 (2008): 2019-29, https://doi.org/10.1016/j.geoforum.2008.07.006; T.C Nzeadibe y H.C. Iwuoha, "Informal waste recycling in Lagos, Nigeria", Communications in Waste \&amp; Resource Management 9 (2008): 24-30; Jutta Gutberlet, "Empowering collective recycling initiatives: Video documentation and action research with a recycling co-op in Brazil”, Resources, Conservation and Recycling 52, no 4 (2008): 659-70, https://doi.org/10.1016/j.resconrec.2007.08.006; Jutta Gutberlet y Angela M. Baeder, "Informal Recycling and Occupational Health in Santo André, Brazil", International Journal of Environmental Health Research 18, no 1 (2008): 1-15, https://doi.org/10.1080/09603120701844258.

12 Asim et al., op.cit.; Eric Binion y JuttaGutberlet. The effects of handling solid waste on the wellbeing of informal and organized recyclers: A review of the literature. International Journal of Occupational and Environmental Health 18, no 1 (2012): 43-52. https://doi.org/10.1179/1077352512Z.0000000001; Emenda Sembiring y Vilas Nitivattananon, "Sustainable solid waste management toward an inclusive society: Integration of the informal sector", Resources, Conservation and Regycling 54, no 11 (2010): 802-9, https://doi.org/10.1016/j.resconrec.2009.12.010; Martin Medina, "Scavenging in America: back to the future?", Resources, Conservation and Recycling 31, no 3 (2001): 229-40, https://doi.org/10.1016/S0921-3449(00)00082-3; Medina (2000), op.cit; Medina (1997), op.cit.

13 Katusiimeh et al., op.cit ; Asim et al. op. cit; Nzeadibe y Ajaero, op.cit; Nzeadibe y Iwuoha, op. cit; Medina (2000), op.cit; Medina (1997) op.cit.
} 
informal, la literatura tradicional no logra explicar por qué algunas personas optan por este tipo de actividades y se resisten a la formalización cuando tienen la oportunidad de elegir. Como muestran estos estudios, los trabajadores informales pueden sentirse satisfechos con su trabajo y desarrollar una identidad positiva en base a él ${ }^{14}$.

¿Cómo explicar este fenómeno? Los estudios empíricos sobre recolectores han respondido esta pregunta ahondando en las explicaciones que dan los propios sujetos estudiados ${ }^{15}$. Sin embargo, poco han dicho respecto a cómo la sociedad participa en el proceso por el cual estos trabajadores dan sentido a su labor. La teoría del reconocimiento social de Honneth ofrece herramientas para avanzar en este último sentido.

El sentido que las personas otorgan a su trabajo y la identidad que construyen en base a él dependen, en parte, del grado en que éstas sienten que su trabajo es reconocido por la sociedad. Honneth plantea que existen tres formas distintas de reconocimiento social que son igualmente necesarias: el amor, el derecho y la estima social ${ }^{16}$. La violación de estas formas implica, consecuentemente, tres formas de desprecio: maltrato, exclusión y humillación. El trabajo ha sido históricamente una de las fuentes fundamentales de la estima social. La sociedad evalúa a los individuos a partir del trabajo que realizan puesto que éste operaría como indicador de su contribución a la reproducción del colectivo:

la estima social de una persona se mide ampliamente por la contribución que aporta a la sociedad en tanto que trabajo formalmente organizado. En lo concerniente a la estima social, las relaciones de reconocimiento están estrechamente ligadas a la repartición y a la organización del trabajo social $^{17}$

Tradicionalmente, la idea de estima social ha estado aparejada a la figura del empleo formal que caracterizó a los Estados de bienestar europeos, es decir, a un empleo estable que garantiza acceso a los servicios básicos y protección social. Las personas obtendrían el reconocimiento de la sociedad y, por ende, construirían una relación armoniosa con sus trabajos y consigo mismas, cuando sus necesidades materiales están cubiertas y su futuro relativamente asegurado. Por esta razón, para algunos autores, la desprotección social que acompaña la implementación del neoliberalismo en el mundo del trabajo sería experimentada como una "descalificación social"18.

El trabajo de los recolectores de basura está lejos de la figura de empleo que acompaña la idea tradicional de estima social. Para comprender por qué, pese a ello éstos pueden otorgar un sentido positivo a su trabajo, resulta útil la distinción que realiza Honneth entre dos formas de reconocimiento: 1) una forma falsa e "ideológica"; y 2) otra "justa" u original. Mientras que la primera se agota en el reconocimiento simbólico que otorga a los trabajadores una imagen positiva de sí mismos, la segunda implica, además, un componente material que se expresa en un cambio de las disposiciones institucionales hacia los

14 Truman G. Packard, "Do Workers in Chile Choose Informal Employment? A Dynamic Analysis of Sector Choice" (The World Bank, 1 de mayo de 2007); Andrew D. Mason et al., "Informalidad : escape y exclusion" (The World Bank, 1 de enero de 2007); William F. Maloney, "Informality Revisited", World Development 32, no 7 (2004): 1159-78; Catalina Amuedo-Dorantes, "Determinants and Poverty Implications of Informal Sector Work in Chile", Economic Development and Cultural Change 52, no 2 (2004): 347-68, https://doi.org/10.1086/380926; J. P. Pérez Sainz, “The New Faces of Informality in Central America”, Journal of Latin American Studies 30, no 01 (1998): 157-79, https://doi.org/10.1017/S0022216X97004938; Alejandro Portes y Saskia Sassen-Koob, "Making it Underground: Comparative Material on the Informal Sector in Western Market Economies", American Journal of Sociology 93, no 1 (1987): 30-61; David M. Blau, "Self-Employment and Self-Selection in Developing Country Labor Markets", Southern Economic Journal 52, no 2 (1985): 351-63, https://doi.org/10.2307/1059621; Katusiimeh et al. op.cit; Nzeadibe y Ajaero, op.cit.

15 Katusiimeh et al. op.cit; Nzeadibe y Ajaero, op. cit.

16 Axel Honneth. La lutte pour la reconnaissance, (París: Cerf, 2010).

17 Axel Honneth. La sociêté du mépris. Vers une nouvelle théorie critique, (París: La découverte, 2008), p.197.

18 Serge Paugam. La disqualification sociale. Essai sur la nouvelle pauvreté (París: PUF, 2009). 
trabajadores, es decir, en mejores condiciones materiales y protección social. En otras palabras, la sociedad ofrece a las personas distintos tipos de reconocimiento, pero no todos tienen el mismo potencial emancipador. Las personas pueden sentirse realizadas, pero este sentimiento no tiene las mismas consecuencias para la persona si no tiene un correlato institucional, como podría suceder en el caso de los recolectores de basura.

Para Honneth, la imagen de "trabajadores-empresarios" difundida a través de la literatura empresarial contemporánea es un ejemplo típico de oferta ideológica de reconocimiento ${ }^{19}$. El discurso del emprendimiento apela a modificar la relación que tienen las personas con su labor, exacerbando los valores de autonomía y autorrealización. El emprendedor idealmente "se hace" y "depende" de sí mismo, no requiere por ende un empleo estable ni de la protección que brinda el Estado. Constituye para el neoliberalismo el modo ideal de contribución a la sociedad, aun cuando invisibiliza el carácter desigualmente distribuido de las condiciones y las consecuencias de la aplicación de este modelo para las personas ${ }^{20}$. La figura del emprendimiento serviría entonces para "[...] integrar a los individuos y grupos sociales en el orden social dominante ofreciéndoles una imagen positiva de sí mismos"21.

Cabe entonces preguntarse por el sentido que los recolectores de basura atribuyen a su labor, qué tipo de reconocimiento está detrás del sentido positivo que le otorgan algunos de ellos, y por qué estos grupos se pliegan a esta oferta. Estas preguntas orientan el trabajo empírico presentado a continuación.

\section{Contexto}

Para estudiar el sentido que los recolectores otorgan a su labor en Chile resulta necesario entender previamente el contexto en que estos trabajadores se desempeñan.

El golpe militar chileno de 1973 puso fin al proyecto socialista liderado por Salvador Allende y desmanteló la oposición política, dejando el camino libre para la implementación de las reformas neoliberales que tendrían impacto en el resto de la región varios años después. Estas reformas afectaron todas las esferas sociales, desde la industria nacional a la educación. La idea central era que el Estado debía limitarse a un rol subsidiario porque el mercado permitía la autorregulación. De esta manera, las empresas públicas fueron privatizadas, se limitó la protección al trabajo formal y se eliminaron las trabas a la iniciativa empresarial, entre otros cambios sustantivos ${ }^{22}$.

La llegada de la democracia en 1990 no trajo los cambios esperados por los grupos menos conformes con la economía de mercado. Los gobiernos de la Concertación de Partidos por la Democracia (19902010) se hicieron cargo de apaciguar las externalidades sociales de las reformas impuestas durante la dictadura, pero no modificaron sustancialmente los pilares de este modelo ${ }^{23}$.

Desde fines de los años ochenta, Chile ha experimentado un crecimiento económico relativamente estable. Sin embargo, éste no ha sido acompañado de políticas que permitan reducir la brecha social existente, ubicando al país en uno de los primeros lugares en el ranking de desigualdad (Programa de las

19 Honneth (2008), op. cit.

${ }^{20}$ Luc Boltanski y Eve Chiapello, Le Nouvel esprit du capitalisme (Paris: Gallimard, 1999).

${ }^{21}$ Honneth (2008), op. cit., p. 245

22 Ricardo Ffrench-Davis, Entre el Neoliberalismo y el Crecimiento con Equidad: Tres Décadas de Política Económica en Chile (Santiago, Chile: Dolmen, 2001).

23 Tomás Moulian, Chile actual: anatomía de un mito (Santiago de Chile: Lom Ediciones, 2002); Manuel Antonio Garretón, Neoliberalismo Corregido Y Progresismo Limitado. Los gobiernos de la Concertación en Chile, 1990-2010, Pensar América Latina (Santiago de Chile: Editorial Arcis-Clacso, 2012). 
Naciones Unidas para el Desarrollo 2017). Expresión de este problema ha sido el peso que sigue ocupando el sector informal en el mercado laboral. Si bien la tasa de informalidad en Chile es menor al promedio de la región (30\% contra 53\% $)^{24}$, demuestra que un tercio de la población se desempeña fuera del marco regulatorio y sin las garantías que, si bien limitadas, ofrece el empleo formal.

En particular, se estima que existen 60,000 personas dedicadas a la recolección informal de basura ${ }^{25}$. Esta ocupación ha experimentado grandes cambios en las últimas décadas, de la mano con las políticas económicas antes señaladas. En efecto, el actual sistema de gestión de la basura tiene su origen en las reformas llevadas a cabo durante el régimen militar ${ }^{26}$. Antes de estas reformas, las municipalidades eran los organismos encargados de la recolección y depósito de la basura. El pago de cada propiedad por el derecho de aseo se calculaba mediante la repartición de los costos del servicio entre todos los habitantes de la comuna. Con la publicación de la Ley Orgánica Constitucional de Municipalidades de 1982, se privatizó la administración del servicio manteniendo el sistema de cobro ${ }^{27}$. Tras el retorno a la democracia, se reconoció este cambio, pero se transfirió a las municipalidades la responsabilidad de fijar el monto anual de los derechos de aseo y contratar, mediante licitaciones, a las empresas para realizar estos servicios (Ley 19 300)28.

Hasta el año 2017, el reciclaje no estaba formalmente incorporado al sistema de gestión de residuos, puesto que no existía una normativa general al respecto. Aunque algunas municipalidades en conjunto con ONGs desarrollaron programas específicos para promover esta práctica, el reciclaje se llevaba a cabo, en gran medida, en un circuito paralelo que incorporaba recolectores informales, intermediarios y empresas recicladoras ${ }^{29}$. En el 2010, se estimaba que los recolectores informales en Chile eran responsables de rescatar aproximadamente el $60 \%$ de los residuos que se reciclaban en el país ${ }^{30}$. A pesar de esto, la tasa de reciclaje bordeaba sólo un 10\%, cinco puntos por debajo del promedio de la región ${ }^{31}$, lo que llevó al Estado a elaborar una nueva política de reciclaje que fue aprobada en el 2016 e implementada en el 2017. La nueva ley instituye un sistema formal de gestión integral de los residuos el cual, si bien incorpora nuevos objetivos, sigue priorizando la responsabilidad del mercado por sobre la del Estado. En efecto, la ley atribuye a los fabricantes de nueve productos definidos como prioritarios ${ }^{32}$

${ }^{24}$ José Manuel Salazar-Xirinachs y Juan Chacaltana. Politicas de formalización en América Latina. Avances y desafíos (Lima: OIT, 2008)

${ }_{25}$ Mesa para la Inclusión de los Recicladores de Base. Políticas para la inclusión de los recicladores de base al sistema de gestión de residuos municipales en Chile. (Santiago: OIT, 2013) https://www.ilo.org/wcmsp5/groups/public/---americas/---ro-lima/---srosantiago/documents/genericdocument/wcms_205403.pdf

26 Alfredo Rodríguez y Paula Rodríguez. Políticas neoliberales en Santiago de Chile: políticas contra la ciudad. Questiones urbano regionales. Revista del instituto de la Ciudad, 2, no 2 (2013), p. 9-31; Giuletta Fadda y Enrique Oviedo. Gestión local de servicios públicos en Chile: aseo, vivienda y educación. Los casos de Talca y Temuco. En: Alfredo Rodríguez y Fabio Velázquez (Eds.), Municipio y servicios públicos: Gobiernos locales en ciudades intermedias de América Latina (pp. 165-187) (Santiago: Ediciones Sur, 1994)

27 Sonia Paecke, La difícil sustentabilidad de una ciudad neoliberal. En: C. De Mattos, M. Ducci, A. Rodríguez, \& G. Yáñez Warner (Eds.), Santiago en la globalización: ¿Una nueva Ciudad? (pp. 189-215). (Santiago: Ediciones Sur, 2004).

28 Juan Pablo Aguilera, Raúl Salazar, Miguel Angel Sepúlveda, Moisés Sepúlveda e Iván Santelices. Residuos sólidos domiciliarios: logística, una herramienta moderna para enfrentar este antiguo problema. Revista Ingeniería Industrial, 5, nº1 (2006), p. 77-87; José Góngora, Beneficios y costos de políticas públicas ambientales en la gestión de residuos sólidos: Chile y Países seleccionados, (Santiago: CEPAL, 2003).

${ }^{29}$ Klaus-Rainer Bräutigam, Tahnee Gonzalez, Marcel Szanto, Helmut Seifert y Joachim Vogdt. Municipal solid waste management in Santiago de Chile: challenges and perspectives towards sustainability. En: D. Heinrichs, K. Krellenberg, B. Hansjürgens, \& F. Martinez (Eds.), Risk Habitat Megacity (pp. 279-301). (Heidelberg: Springer, 2012).

30 Mesa para la Inclusión de los Recicladores de Base, op.cit.

31 Comisión Nacional del Medio Ambiente. Primer Reporte de Manejo de Residuos Sólidos en Cbile. Santiago de Chile: CONAMA, 2010

32 Los nueve productos definidos como prioritarios son los aceites lubricantes; aparatos eléctricos y electrónicos (celulares, ampolletas, monitores, hornos microondas, refrigeradores, etc.); diarios, periódicos y revistas; envases y embalajes (de vidrio, plástico, papel, cartón, metal y madera); medicamentos; neumáticos; pilas y baterías; plaguicidas; y vehículos. 
la responsabilidad de organizar y financiar el sistema, incluyendo el cumplimiento de las metas de reciclaje que serán fijadas por el Ministerio del Medio Ambiente. El rol de las municipalidades se limita a crear convenios para facilitar esta tarea e incorporar en la ordenanza municipal la obligación de recolección separada de estos productos. Asimismo, la ley crea un sistema nacional de certificación y registro de los recolectores informales, lo que les permitirá formar parte del circuito. De esta manera, la nueva normativa institucionaliza la idea que los recolectores de basura son trabajadores independientes o pequeñas empresas que deben competir en el mercado por los recursos, con la ayuda de las herramientas que les entrega la certificación. El "Movimiento Nacional de Recicladores" (MNR), organización que representa formalmente a los recolectores informales a nivel nacional, respaldó la nueva normativa.

\section{Metodología}

Para responder las preguntas que orientan este artículo, partimos analizando los resultados de la "Primera Encuesta sobre Condiciones Socio-laborales de Recicladore(a)s" (PECR) realizada por un equipo de investigación y Fundación Casa de La Paz. La PECR incorpora una centena de preguntas para describir el trabajo y el perfil de los recolectores, incluyendo la percepción que tienen de su empleo. Fue aplicada principalmente en cuatro comunas de la ciudad de Santiago: Peñalolén, Recoleta, Quinta Normal y Santiago. Estas comunas se eligieron considerando la existencia de recicladores, el apoyo del municipio al proyecto y el hecho de no contar con iniciativas de valorización de los recicladores en curso. En total, se encuestó a 404 recicladores informales, hombres y mujeres, contactados a partir de una técnica no sistemática de barrido de casos, es decir, a través del contacto directo que establecieron los encuestadores con los recicladores que encontraron en distintos sectores de las comunas señaladas. La Tabla 1 resume algunas características de la muestra. Si bien la PECR no es estadísticamente representativa -no existía hasta la fecha en Chile un catastro oficial del total de recicladores de residuos-, sigue siendo la fuente más completa de información disponible sobre este tipo de actividad.

Tabla 1: Descripción de la muestra por sexo y comuna donde se realizó encuesta

\begin{tabular}{|c|c|c|c|c|}
\hline & $\mathbf{N}$ & Media & Mínimo & Máximo \\
\hline \multirow[t]{2}{*}{ Edad } & 398 & 48 & 18 & 78 \\
\hline & Categoría & $\mathbf{N}$ & $\%$ & \\
\hline \multirow[t]{2}{*}{ Sexo } & Mujer & 179 & 44,3 & \\
\hline & Hombre & 225 & 55,7 & \\
\hline \multirow[t]{5}{*}{ Comuna } & Peñalolén & 145 & 35,9 & \\
\hline & Quinta Normal & 49 & 12,1 & \\
\hline & Recoleta & 122 & 30,2 & \\
\hline & Santiago & 84 & 20,8 & \\
\hline & Sin información & 4 & 1 & \\
\hline \multirow{6}{*}{$\begin{array}{l}\text { Nivel } \\
\text { educacional }\end{array}$} & Sin estudios & 24 & 5,9 & \\
\hline & $\begin{array}{l}\text { Básica } \\
\text { incompleta }\end{array}$ & 135 & 33,4 & \\
\hline & Básica completa & 82 & 20,2 & \\
\hline & $\begin{array}{l}\text { Media } \\
\text { incompleta }\end{array}$ & 80 & 19,8 & \\
\hline & Media completa & 61 & 15,1 & \\
\hline & $\begin{array}{l}\text { Técnica } \\
\text { superior } \\
\text { incompleta }\end{array}$ & 3 & 0,7 & \\
\hline
\end{tabular}




\begin{tabular}{|l|l|c|c|}
\hline & $\begin{array}{l}\text { Técnica } \\
\text { superior } \\
\text { completa }\end{array}$ & 8 & 2 \\
\cline { 2 - 4 } & $\begin{array}{l}\text { Universitaria } \\
\text { incompleta }\end{array}$ & 2 & 0,5 \\
\cline { 2 - 4 } & Perdido & 9 & 2,2 \\
\hline
\end{tabular}

Fuente: Elaboración propia

Para identificar eventuales diferencias dentro del grupo de trabajadores estudiados, utilizamos la técnica de cluster en dos fases o bietápico. Utilizamos esta técnica porque a diferencia de otros análisis de cluster no jerárquico o jerárquico permite determinar automáticamente el número óptimo de conglomerados y crear modelos de conglomerados con variables categóricas y continuas. Como principal criterio de agrupación, incluimos la variable "satisfacción general respecto al empleo". Este indicador corresponde al puntaje obtenido por los encuestados, en una escala de 1 a 20 , donde 1 es muy insatisfecho y 20 muy satisfecho ${ }^{33}$. El indicador se construyó a partir de dos preguntas de la encuesta donde se interrogaba a los encuestados por los aspectos positivos y negativos del trabajo, partiendo de una lista preestablecida de elementos (la lista era idéntica en ambas preguntas). El puntaje asignado a cada sujeto se calculó sumando todos los aspectos que destacaba de su trabajo, menos los aspectos que criticaba, y estandarizando este resultado a una escala con los parámetros señalados.

En la construcción de la tipología, se incluyen además seis variables que miden distintos aspectos objetivos del trabajo. De esta manera, se busca que la tipología no refleje sólo las diferentes percepciones, sino la pluralidad de relaciones que tienen los recolectores con su trabajo.

En específico, las variables consideradas son:

- Capacidad de organización: variable categórica donde 0 indica que la persona no ha participado ni participa en alguna organización de recicladores y 1 que dicho sujeto sí cumple con estas condiciones. La variable fue creada a partir de las preguntas "¿No participa en ninguna asociación de recicladores, o agrupación gremial de recicladores u organización comunitaria de recicladores?" y "Y anteriormente, ¿usted participó en alguna organización o asociación de reciclaje?")

- Plan de salud: variable categórica donde 0 indica que la persona no está inscrita en el sistema de salud y 1 que sí cumple con esta condición. ${ }^{34}$

- Intensidad de trabajo: variable continua que indica el promedio de horas totales trabajadas partido por el total de días efectivamente trabajados.

- Ingreso promedio mensual declarado: variable continua que indica la respuesta de los encuestados a la pregunta "Más o menos (en promedio), ¿cuánto gana usted en el trabajo del reciclaje al mes?"

- Seguridad e higiene: variable categórica dicotómica donde 0 indica que el encuestado no ha presentado una enfermedad asociada a la actividad durante los últimos 6 meses anteriores a la encuesta y 1 que sí cumple con esta condición ${ }^{35}$

- Suficiencia del empleo: variable nominal dicotómica donde 0 indica que la persona no tiene otro empleo paralelo y 1 que sí cumple con esta condición.

\footnotetext{
$33 \mathrm{El}$ puntaje se calcula sumando todos los aspectos que los participantes destacan de su trabajo, menos los aspectos que critican, y estandarizando este resultado a una escala con los parámetros señalados.

34 En Chile, además de los seguros de salud privado, existe un seguro público que cubre también a quienes son catalogados como "indigentes". Por esta razón, como se verá, gran parte de la muestra está cubierto por un plan de salud.

35 En el proceso de construcción de la tipología, se testeó también la variable "accidente laboral". Sin embargo, esta variable no diferenciaba de manera significativa grupos.
} 
La estadística descritiva de todas estas variables se muestra en la Tabla 2

Tabla 2: Estadística descriptivas variables conglomerados

\begin{tabular}{|c|c|c|c|c|c|}
\hline & $\begin{array}{l}\text { Casos } \\
\text { válidos }{ }^{36}\end{array}$ & Mínimo & Máximo & Media & Desv. típ \\
\hline Satisfacción & 404 & 5 & 19 & 13,4 & 2,5 \\
\hline Intensidad de trabajo & 404 & 2,67 & 17 & 7,2 & 2,2 \\
\hline \multirow{2}{*}{ Ingreso (Pesos chilenos \$) } & 366 & 2000 & 1000000 & 169554,6 & 122331,4 \\
\hline & $\begin{array}{l}\text { Casos } \\
\text { válidos }\end{array}$ & No & Sí & & \\
\hline Otro trabajo & 394 & $298(73,8 \%)$ & $96(23,8 \%)$ & & \\
\hline Enfermedades & 402 & $234(57,9 \%)$ & $168(41,6 \%)$ & & \\
\hline Plan de salud & 402 & $70(17,3 \%)$ & $332(82,2 \%)$ & & \\
\hline Organización & 404 & $326(80,7 \%)$ & $78(19,3 \%)$ & & \\
\hline
\end{tabular}

Adicionalmente, se analizó la distribución social de los tipos construidos, describiendo el promedio de edad y años de trayectoria de cada perfil, así como la presencia de cada tipo en subgrupos definidos por el sexo y el nivel educacional. En los estudios realizados en otros contextos, se ha evidenciado que estos criterios generan diferencias en el nivel de ingreso o las condiciones en que los recicladores informales desempeñan su labor ${ }^{37}$. Esto también podría tener un correlato en el plano subjetivo.

Como complemento al análisis estadístico, en una segunda etapa de la investigación, realizamos 14 entrevistas en profundidad a distintos recolectores encuestados. Buscamos representatividad de los perfiles identificados. El corpus estuvo compuesto por 7 hombres y 7 mujeres, menores de 67 años. Las entrevistas fueron transcritas integralmente y analizadas por codificación abierta.

\section{Resultados}

\section{Las relaciones de los recolectores con su trabajo}

El análisis de cluster en dos fases arrojó como resultado óptimo la existencia de cinco perfiles de recolectores de basura según la relación que tienen con su trabajo. La Tabla 3 muestra las principales características de estos grupos.

Tabla 3. Perfiles de recolectores informales

\begin{tabular}{|l|l|l|l|l|l|}
\hline & Perplejos & Adaptativos & Confiados & Distantes & Conformistas \\
\hline $\mathbf{N}^{\mathbf{o}}$ casos & 99 & 37 & 37 & 78 & 95 \\
\hline Tamaño (\%) & 28.6 & 10.7 & 10.7 & 22.5 & 27.5 \\
\hline Satisfacción & $\mathbf{1 2 . 7 3}$ & 14.62 & $\mathbf{1 5 . 1 9}$ & 13.40 & 13.49 \\
\hline Otro trabajo & $\mathrm{No}(100 \%)$ & $\mathrm{No}(78.4 \%)$ & $\mathrm{No}(100 \%)$ & $\mathrm{Si}(\mathbf{1 0 0} \%)$ & $\mathrm{No}(100 \%)$ \\
\hline Enfermedades & $\mathrm{Si}(\mathbf{1 0 0} \%)$ & $\mathrm{No}(100 \%)$ & $\mathrm{No}(100 \%)$ & $\mathrm{No}(61.5 \%)$ & $\mathrm{No}(100 \%)$ \\
\hline
\end{tabular}

36 Se excluyen los casos perdidos (datos faltantes o sin respuesta).

${ }_{37}$ Mitchell, op.cit.; Katusiimeh et al., op.cit.; Do Carmo et al. op.cit; Gutberlet, op.cit; Wilson et al (2006), op.cit; Medina (2000), op. cit.; Medina (1997), op. cit. 


\begin{tabular}{|l|l|l|l|l|l|}
\hline Plan de salud & $\mathrm{Si}(88.9 \%)$ & $\mathrm{No}(\mathbf{1 0 0} \%)$ & $\mathrm{Si}(62.2)$ & $\mathrm{Si}(96.2 \%)$ & $\mathrm{Si}(100 \%)$ \\
\hline Organización & $\mathrm{No}(79.8 \%)$ & $\mathrm{No}(100 \%)$ & $\mathrm{Si}(\mathbf{1 0 0} \%)$ & $\mathrm{No}(83.3 \%)$ & $\mathrm{No}(100 \%)$ \\
\hline Intensidad de trabajo & 7.36 & $\mathbf{8 . 0 1}$ & 7.67 & $\mathbf{6 . 4 6}$ & 7.45 \\
\hline $\begin{array}{l}\text { Ingreso (Pesos chilenos } \\
\mathbf{\$ )}\end{array}$ & $\mathbf{1 5 8 , 0 3 0}$ & 159,459 & $\mathbf{2 1 9 , 0 0 0}$ & $\mathbf{1 3 8 , 6 1 5}$ & 163,736 \\
\hline
\end{tabular}

Fuente: Elaboración propia en base a resultados del análisis de cluster bietápico. Se resaltan en negro los valores extremos por variable. El ingreso en el grupo de "perplejos" está subrayado por ser el valor más bajo de los grupos que se dedican exclusivamente a la actividad de recolección de residuos.

El grupo de los recicladores "perplejos" representa al 28,6\% de los casos. Es el que presenta una postura más crítica respecto de su labor, con una satisfacción de 12,73, aunque por sobre el nivel considerado como el límite entre la satisfacción y la insatisfacción. Esto coincide con el hecho de que este grupo posee las condiciones laborales más desventajosas dentro de la muestra, si se considera como criterio el número de aspectos negativos acumulados: tiene historial de enfermedades laborales, ausencia de organización y el más bajo nivel de ingreso dentro de los recicladores que se dedican exclusivamente a esta actividad. Sin embargo, los individuos clasificados en este grupo están cubiertos por un plan de salud -generalmente FONASA ${ }^{38}-$, y su intensidad de trabajo es la más baja del total de recicladores que se dedican de manera exclusiva a esta actividad.

El segundo grupo de los “adaptativos" representa el 10,7\% de la muestra. Tiene el segundo nivel más alto de satisfacción aun cuando las condiciones de su trabajo son casi tan malas como las del primer grupo. En efecto, no se encuentran organizados, no están cubiertos por un plan de salud, y cuentan con el más alto grado de intensidad de trabajo. Este último aspecto los diferencia de los "conformistas" y puede ser interpretado como el resultado de la búsqueda individual de estos recolectores por mejorar su situación, adaptándose a las condiciones adversas más que cuestionándolas directamente.

El tercer grupo de los "distantes" (el 22,5\% de la muestra) está compuesto por aquellos recolectores que no se dedican exclusivamente a la recolección de residuos. Su grado de satisfacción con la actividad de reciclaje no es muy alto (es el segundo más bajo de la muestra), lo que sugiere una relación más indiferente con el oficio, y puede relacionarse con el hecho de que realizan otras actividades remuneradas en paralelo.

El cuarto grupo es representado por la categoría de los "conformistas" (27,5\% de la muestra), la cual agrupa a los individuos que se dedican exclusivamente a la recolección de residuos y cuyas condiciones laborales son de calidad intermedia. Su nivel de satisfacción es más alto que los "perplejos" y los "distantes", aun cuando tienen condiciones laborales similarmente negativas, como un sueldo menor al mínimo nacional y la falta de organización. Por esta razón, hablamos de "conformistas", en el sentido de que no parecen cuestionar su posición marginal dentro del grupo de recolectores, pero tampoco intensificar su trabajo para superar su situación como los "adaptativos".

Finalmente, identificamos el grupo de los "confiados", que equivale al 10,7\% de los encuestados. Es la figura antagónica de los "perplejos" pues sus condiciones laborales son las mejores dentro de la muestra, aunque igualmente precarias en relación a otros empleos formales. Los recolectores clasificados en este grupo son los únicos que han participado o participan en alguna organización y alcanzan el más alto grado de satisfacción con el empleo. Esto sugiere que son los más identificados con su labor y su grupo de referencia, lo que coincide con el hecho de que presentan el más alto ingreso (levemente superior al

38 Fonasa es la sigla correspondiente al Fondo Nacional de Salud, ente financiero encargado de recaudar, administrar y distribuir los dineros estatales dedicados a la salud en Chile. Un poco más del $80 \%$ de la población accede a la salud a través de este sistema. 
sueldo mínimo en Chile ${ }^{39}$ ). La intensidad de trabajo es, sin embargo, el costo de este relativo "éxito económico" pues los individuos trabajan más horas que el resto.

Esta tipología nos entrega una imagen más compleja de la relación de los recolectores con su trabajo. El grado de satisfacción varía en función de las condiciones objetivas de la actividad, aunque no linealmente. Sin embargo, sorprende que todos los grupos muestren algún grado de satisfacción con su trabajo pese a que las condiciones objetivas son bastante precarias.

La Tabla 4 muestra la distribución de los perfiles en la muestra encuestada. Se confirma la importancia que tiene el género en la relación de los recolectores con su trabajo. Los grupos "perplejos" y "conformistas" son significativamente más feminizados que el grupo de los "adaptativos". En efecto, las mujeres representan el $47 \%$ y el $48 \%$ del total de personas clasificadas en cada uno de estos grupos; el $32 \%$ y $31,3 \%$ respectivamente del total de mujeres de la muestra; y el 13,9\% y 13,6\% del total de participantes del estudio. Si bien entre los "confiados" existe una proporción mayor de hombres que de mujeres, la diferencia no es estadísticamente relevante.

Tabla 4. Variables asociadas a tipología de recicladores

\begin{tabular}{|c|c|c|c|c|c|c|c|}
\hline & & \multicolumn{5}{|c|}{ Tipología Condiciones Laborales } \\
\hline & & & $\begin{array}{l}\text { Adaptativo } \\
\text { (A) }\end{array}$ & $\begin{array}{l}\text { Confiado } \\
\text { (B) }\end{array}$ & $\begin{array}{l}\text { Distante } \\
\text { (C) }\end{array}$ & $\begin{array}{l}\text { Conformist } \\
\text { a } \\
\text { (D) }\end{array}$ & $\begin{array}{l}\text { Perplejo } \\
\text { (E) }\end{array}$ \\
\hline \multicolumn{2}{|c|}{ Años en la actividad } & Media & 15 & $18(\mathrm{C})^{*}$ & 11 & $16(\mathrm{C})^{*}$ & $16(\mathrm{C})^{*}$ \\
\hline \multicolumn{2}{|l|}{ Edad } & Media & $52,30(\mathrm{C})^{*}$ & 48,41 & 44,33 & 49,04 & $\begin{array}{l}50,19 \\
(\mathrm{C})^{*}\end{array}$ \\
\hline \multirow{8}{*}{ Sexo } & \multirow{4}{*}{ Mujer } & Recuento & 8 & 14 & 33 & $47(\mathrm{~A})^{*}$ & $48(\mathrm{~A})^{*}$ \\
\hline & & $\%$ dentro de sexo & $5,3 \%$ & $9,3 \%$ & $22 \%$ & $31,3 \%$ & $32 \%$ \\
\hline & & $\begin{array}{l}\% \text { dentro de } \\
\text { tipología }\end{array}$ & $21,6 \%$ & $37,8 \%$ & $42,3 \%$ & $49,5 \%$ & $48,5 \%$ \\
\hline & & $\%$ del total & $2,3 \%$ & $4 \%$ & $9,5 \%$ & $13,6 \%$ & $13,9 \%$ \\
\hline & \multirow{4}{*}{ Hombre } & Recuento & $29(\mathrm{D} \mathrm{E})^{*}$ & 23 & 45 & 48 & 51 \\
\hline & & $\%$ dentro de sexo & $14,8 \%$ & $11,7 \%$ & $23 \%$ & $24,5 \%$ & $26 \%$ \\
\hline & & $\begin{array}{l}\% \text { dentro de } \\
\text { tipología }\end{array}$ & $78,4 \%$ & $62,2 \%$ & $57,7 \%$ & $50,5 \%$ & $51,5 \%$ \\
\hline & & $\%$ del total & $8,4 \%$ & $6,6 \%$ & $13 \%$ & $13,9 \%$ & $14,7 \%$ \\
\hline \multirow{7}{*}{$\begin{array}{l}\text { Nivel } \\
\text { educacio } \\
\text { nal (NE) }\end{array}$} & \multirow{4}{*}{ Sin estudios } & Recuento & 3 & 1 & 3 & 2 & 10 \\
\hline & & $\%$ dentro de NE & $15,8 \%$ & $5,3 \%$ & $15,8 \%$ & $10,5 \%$ & $52,6 \%$ \\
\hline & & $\begin{array}{l}\% \text { dentro de } \\
\text { tipología }\end{array}$ & $8,3 \%$ & $2,7 \%$ & $3,9 \%$ & $2,2 \%$ & $10,2 \%$ \\
\hline & & $\%$ del total & $0,9 \%$ & $0,3 \%$ & $0,9 \%$ & $0,6 \%$ & $2,9 \%$ \\
\hline & \multirow{3}{*}{ Básica incompleta } & Recuento & 15 & 15 & 21 & 31 & 35 \\
\hline & & $\%$ dentro de NE & $12,8 \%$ & $12,8 \%$ & $17,9 \%$ & $26,5 \%$ & $29,9 \%$ \\
\hline & & $\begin{array}{l}\% \text { dentro de } \\
\text { tipología }\end{array}$ & $41,7 \%$ & $40,5 \%$ & $27,6 \%$ & $33,3 \%$ & $35,7 \%$ \\
\hline
\end{tabular}

${ }^{39}$ El sueldo mínimo fue de PCH $\$ 193,000$ (alrededor de 320 dólares) durante el 2013, fecha en que se levantaron los datos. 


\begin{tabular}{|c|c|c|c|c|c|c|}
\hline & $\%$ del total & $4,4 \%$ & $4,4 \%$ & $6,2 \%$ & $9,1 \%$ & $10,3 \%$ \\
\hline \multirow{4}{*}{ Básica completa } & Recuento & 8 & 8 & 14 & 26 & 19 \\
\hline & $\%$ dentro de NE & $10,7 \%$ & $10,7 \%$ & $18,7 \%$ & $34,7 \%$ & $25,3 \%$ \\
\hline & $\begin{array}{l}\% \text { dentro de } \\
\text { tipología }\end{array}$ & $22,2 \%$ & $21,6 \%$ & $18,4 \%$ & $28,0 \%$ & $19,4 \%$ \\
\hline & $\%$ del total & $2,4 \%$ & $2,4 \%$ & $4,1 \%$ & $7,6 \%$ & $5,6 \%$ \\
\hline \multirow{4}{*}{ Media incompleta } & Recuento & 5 & 6 & 17 & 17 & 20 \\
\hline & $\%$ dentro de $\mathrm{NE}$ & $7,7 \%$ & $9,2 \%$ & $26,2 \%$ & $26,2 \%$ & $30,8 \%$ \\
\hline & $\begin{array}{l}\% \text { dentro de } \\
\text { tipología }\end{array}$ & $13,9 \%$ & $16,2 \%$ & $22,4 \%$ & $18,3 \%$ & $20,4 \%$ \\
\hline & $\%$ del total & $1,5 \%$ & $1,8 \%$ & $5 \%$ & $5 \%$ & $5,9 \%$ \\
\hline \multirow{4}{*}{ Media completa } & Recuento & 3 & 7 & 15 & 15 & 13 \\
\hline & $\%$ dentro de NE & $5,5 \%$ & $13,2 \%$ & $28,3 \%$ & $28,3 \%$ & $24,5 \%$ \\
\hline & $\begin{array}{l}\% \text { dentro de } \\
\text { tipología }\end{array}$ & $8,3 \%$ & $18,9 \%$ & $19,7 \%$ & $16,1 \%$ & $13,3 \%$ \\
\hline & $\%$ del total & $0,9 \%$ & $2,1 \%$ & $4,4 \%$ & $4,4 \%$ & $3,88 \%$ \\
\hline \multirow{4}{*}{$\begin{array}{l}\text { Técnica } \\
\text { incompleta }\end{array}$} & Recuento & $0 * *$ & $0 * *$ & 2 & $0 * *$ & $0 * *$ \\
\hline & $\%$ dentro de $\mathrm{NE}$ & $0,0 \%$ & $0 \%$ & $100 \%$ & $0 \%$ & $0 \%$ \\
\hline & $\begin{array}{l}\% \text { dentro de } \\
\text { tipología }\end{array}$ & $0,0 \%$ & $0 \%$ & $2,6 \%$ & $0 \%$ & $0 \%$ \\
\hline & $\%$ del total & $0,0 \%$ & $0 \%$ & $0,6 \%$ & $0 \%$ & $0 \%$ \\
\hline \multirow{4}{*}{ Técnica completa } & Recuento & 2 & $0 * *$ & 3 & 2 & 1 \\
\hline & $\%$ dentro de NE & $25 \%$ & $0 \%$ & $37,5 \%$ & $25 \%$ & $12,5 \%$ \\
\hline & $\begin{array}{ll}\% & \text { dentro } \\
\text { tipología } & \\
\end{array}$ & $5,6 \%$ & $0 \%$ & $3,9 \%$ & $2,2 \%$ & $1 \%$ \\
\hline & $\%$ del total & $0,6 \%$ & $0 \%$ & $0,9 \%$ & $0,6 \%$ & $0,3 \%$ \\
\hline \multirow{4}{*}{$\begin{array}{l}\text { Universitaria } \\
\text { incompleta }\end{array}$} & Recuento & 0 & $0^{* *}$ & 1 & 0 & $0^{* *}$ \\
\hline & $\%$ dentro de $\mathrm{NE}$ & $0 \%$ & $0 \%$ & $100 \%$ & $0 \%$ & $0 \%$ \\
\hline & $\begin{array}{l}\% \text { dentro de } \\
\text { tipología }\end{array}$ & $0 \%$ & $0 \%$ & $1,3 \%$ & $0 \%$ & $0 \%$ \\
\hline & $\%$ del total & $0 \%$ & $0 \%$ & $0,3 \%$ & $0 \%$ & $0 \%$ \\
\hline
\end{tabular}

Joración propia en base a resultados de comparación de proporciones y medias de columna. Los resultados se basan bilaterales con un nivel de significación 0.05 . Se señala con un asterisco $\left(^{*}\right)$ los casos donde existe una asociación entre las variables. Se indica con dos asteriscos $\left(^{* *}\right)$ los casos donde no existen casos suficientes para realizar la lística.

y el tiempo que se lleva reciclando son también elementos que diferencian a los perfiles los. Los "distantes" son significativamente más jóvenes y cuentan con menos años de a que las otras categorías. En otras palabras, para quienes están al inicio de su carrera, la in de basura pareciera ser más una forma complementaria de subsistencia que un oficio con el ntifican. Los "confiados" son los que más años llevan reciclando (18 años) y los más satisfechos ıbajo: los "adaptativos" y "perplejos" también presentan un promedio de años relativamente .5 y 16 años) y, sin embargo, tienen una identificación más problemática con su labor. El nivel al no mostró una asociación significativa con la tipología obtenida. Sin embargo, hay que señalar yor parte de la muestra no alcanzó a terminar la enseñanza media. 


\section{El reconocimiento simbólico de los recolectores de basura}

La satisfacción de los recolectores con su trabajo sugiere que las condiciones precarias en que desempeñan esta labor no les impide construir una imagen positiva de sí mismos. Las entrevistas confirman este fenómeno y muestran que este posicionamiento subjetivo tiene relación con la identificación de los recolectores a la figura del "emprendedor".

Independiente del grupo al que pertenecen, los recolectores entrevistados ven su labor más como un "negocio" que como una ocupación precaria y recurren a un lenguaje gerencial para referirse a él. A través de la identificación con el "emprendedor", los recolectores resaltan su capacidad individual de sobreponerse a sus condiciones de origen y abrazan la esperanza de una movilidad social ascendente. El "emprendimiento" aparece como un medio que "iguala" en el plano simbólico a los recolectores con otras categorías sociales, en la medida en que los vuelve individuos potencialmente competentes para tener éxito económico ${ }^{40}$.

Si bien la identificación con el "emprendedor" es más evidente en el grupo de los “confiados" se observa también en los otros grupos. La mayor parte de los entrevistados reconoce en efecto las habilidades "empresariales" como necesarias para su labor e intentan seguir el ejemplo de los "confiados":

(...) hay gallos ${ }^{41}$ que andan comprando pa' revender que ya tienen auto camioneta po. No son tontos, las hermosean las cosas. [Por ejemplo] Los mismos gallos que compran zapatillas pa' revender, las lavan [primero]. Tú se las vendí en 2 lucas $^{42}$ y ellos piden 17 lucas, 8 lucas [después], porque las revenden, ¿me entiende o no? Eso debería hacer yo, porque [ahora] yo voy saco las cosas y las tiro baratas. Y yo digo, ¿por qué no soy más sabia y la hago yo esa pega ${ }^{43}$ ? Si las dejo más bonitas gano más plata. (Marcia, "Conformista", 69 años)

La precariedad material que caracteriza a la recolección de basura hace que la identificación con la figura de emprendedor permanezca tensionada e imperfecta, sobre todo en los "perplejos", "adaptativos", "distantes" y "conformistas", quienes padecen las situaciones más extremas. Así, por ejemplo, pese a la satisfacción que experimentan, la gran mayoría de los entrevistados percibe que esta labor se ha vuelto más difícil con los años. No sólo porque se han multiplicado las campañas privadas en centros comerciales o espacios públicos para recolectar desechos que se asocian directamente con las empresas de reciclaje prescindiendo de los recolectores informales, sino también porque han cambiado los patrones de conducta de la gente. Así por ejemplo, Marcia cuenta:

En el tiempo en que yo cachureaba ${ }^{44}$ me acuerdo yo que traía lámparas antiguas, yo traía cachureos buenos y en un ratito me hacía, me acuerdo, 60 lucas. ¡En un ratito! Pero ahora no po. Ahora la gente vende las cosas, muy poca gente da las cosas como antes. A mí [antes] me llenaban el triciclo con cosas, ahora la gente las sale a vender (...) (Marcia, "Conformista", 69 años).

Los recolectores definen las condiciones del éxito y la posibilidad de surgir económicamente en este nuevo contexto en términos empresariales, enfatizando el rol individual en el proceso. Por un lado, los

\footnotetext{
40 Gustavo Sánchez. Los efectos del afecto: un análisis posfundacional del discurso del emprendimiento de la Corfo. Tesis para optar al grado de Magíster en Sociología de la modernización, Facultad de Ciencias Sociales, Universidad de Chile, 2018

${ }^{41}$ Hombres.

42 "Luca" significa mil pesos chilenos en la jerga popular.

43 "Pega" significa trabajo en la jerga popular.

44 "Cachurear" refiere a buscar desechos aptos para ser reciclados.
} 
entrevistados señalan que se debe ser "pillo" o "busquilla"45 para identificar las nuevas demandas y agregar constantemente más valor a los objetos recolectados con el fin de venderlos más caros. Como señala David ("Confiado", 49 años), se trata de ser capaz de "descubrir negocios nuevos, nichos nuevos" y aprovechar los "mercados temporales". Las entrevistas están llenas de referencias a este tipo de soluciones individuales: inventar un nuevo material que puede reciclarse, arreglar los objetos recolectados antes de venderlos, vender separadamente las piezas de un objeto, etc. Todas estas acciones permitirían a los recolectores enfrentar los cambios que han afectado su labor. Por otro lado, los entrevistados destacan la importancia de las habilidades individuales. La posibilidad de aumentar los ingresos dependería de: a) la capacidad de los recicladores de "generar confianza" y un "buen trato" con quienes pueden entregarles objetos para reciclar; b) saber negociar con los intermediarios para maximizar las ganancias; c) generar una "clientela" más o menos estable para garantizar la venta de los objetos que se ofrecen directamente al público.

La contracara de la identificación de los recolectores a la figura del emprendedor es el rechazo que presentan respecto de la acción colectiva. Con excepción de los "confiados", los recolectores desconfían de esta forma de acción, lo que podría explicar el bajo nivel de afiliación a organizaciones de recolectores (solo el 10\%, según la encuesta). José, dirigente de una de estas organizaciones, bien lo señala: la tentativa de organizar a los recicladores ha chocado con el "individualismo" de la mayor parte de ellos. El rechazo a la acción colectiva resulta paradojal si se considera que los afiliados a este tipo de organización gozan de mejores ingresos que el resto de los recolectores (PECR). Como señala el dirigente citado anteriormente, la organización ha permitido a los recicladores negociar en mejores condiciones con las empresas de reciclaje o generar alianzas con entidades públicas y privadas. En este sentido, el rechazo a la acción colectiva no responde a un cálculo instrumental. Más bien parece tratarse de una postura normativa alimentada por la retórica imperante del "emprendedor".

Siguiendo a Honneth, es posible interpretar esta identificación con la figura del emprendedor como la aceptación de una forma ideológica de reconocimiento. La auto-estima de los recolectores reposaría así en el reconocimiento netamente simbólico que hace la sociedad de su trabajo. La entrevista sugiere que los recolectores se pliegan a esta oferta precaria de reconocimiento, en parte, porque sus expectativas están limitadas por la posición que ocupan en el espacio social.

Como se evidenció a través de la encuesta, quienes se desempeñan como recolectores provienen de la parte inferior de la estructura social. Por esta razón, tienden a valorar su trabajo en relación a otras actividades a las que pueden o han podido acceder, esto es comparándolo con experiencias laborales anteriores. Éstas han sido a menudo marcadas por la discriminación y el desprecio. En efecto, varios de los entrevistados conocieron al menos un empleo caracterizado por la precariedad extrema y el abuso antes de comenzar con el reciclaje. Los empleos sin contrato, largas jornadas de trabajo, escaso ingreso y malos tratos por parte de la jerarquía, parecen ser la regla en la trayectoria profesional de este grupo social. Así, por ejemplo, Mariela ("Distante", 28 años) recuerda cómo la dueña de la casa donde trabajaba antes de comenzar a reciclar, la maltrataba diciéndole que "las nanas ${ }^{46}$ son gatos de la casa, comen lo que sobra".

La subordinación salarial expone a las personas de la condición social de estos recolectores a toda la fuerza de la violencia y la discriminación dominante en el mercado laboral chileno, lo que se acentúa en el caso de las mujeres. Por contraste con este tipo de empleo, la recolección informal de basura aparece como un espacio de autonomía liberadora. Al otorgarles la posibilidad de controlar sus tiempos, sus espacios y su contacto con otros, la actividad de recolección permite a estos individuos eludir los

\footnotetext{
45 Dos formas coloquiales para referirse al hecho de ser astuto.

46 Trabajadoras de casa particular.
} 
vejámenes que su grupo social experimenta a menudo cuando está sometido a las exigencias del trabajo asalariado. Por esto el "No tener jefe" y "Tener sus propios horarios" son los aspectos de la labor de reciclador más apreciados en todos los grupos (PECR). Los recicladores viven el hecho de "que nadie los esté mandando" (Eraclio, "Confiado", 72 años) como una forma de autonomía. En este espacio liberado, resulta más fácil construir la confianza, el respeto y la estima personal.

Los recicladores también valoran su labor por contraste con los otros oficios que son accesibles a personas de sus características sociales. Los entrevistados son conscientes de que, dado su bajo capital cultural tanto como social, existen pocas posibilidades de acceder a un empleo mejor que el de recolector de basura. Esto resulta particularmente evidente en el caso de quienes han pasado por la cárcel. Como lo señala Mauricio ("Perplejo", 43 años), quien comenzó a trabajar en esta actividad luego de 25 años de prisión: "Me gusta recolectar. Hay trabajo para todos y uno se puede ganar la vida honradamente". Para las personas como Mauricio, reinsertarse y permanecer en el mercado laboral constituye un desafío difícil. La estigmatización negativa que pesa sobre los ex presidiarios bloquea generalmente su movilidad social y dificulta el acceso al empleo. En comparación con las alternativas que el mercado laboral chileno ofrece, la recolección de basura aparece a estos sujetos como una actividad democrática donde uno puede trabajar libre de prejuicios y reencontrar la dignidad.

La recolección de basura puede también cobrar sentido no solo por contraste con otras opciones laborales, sino también porque representa una salida a una situación familiar vivida como agobiante. Así, por ejemplo, María Elena ("Adaptativa", 58 años) comenzó a trabajar incursionando en el mundo del reciclaje tras diagnosticarse un cáncer terminal a su marido. Luego de una primera etapa de "vergüenza y asco", confiesa haber logrado sentirse "orgullosa" y "respetada" pues logró una independencia económica antes impensable. La satisfacción de haber accedido a algo que en el antiguo régimen familiar resultaba imposible, compensa el malestar producido por los escasos ingresos percibidos. En este caso, la dominación de género se agrega a la dominación de clase que pesa sobre el conjunto de los entrevistados:

(...) Mire sabe que los pesitos que salgan 3 mil, 3 mil, lo que haga en el día es plata que yo tengo para mí, es plata que yo no tengo que andar pidiendo, porque después si no tengo pa' devolver y no me gusta quedar por sinvergüenza como se dice que después no pague, entonces tengo mis pesitos y ahí sí me alcanza como le digo yo a veces pa' carne si no no po, me hago unos fideos con, qué sé yo, hasta con huevitos revueltos (María Elena, "Adaptativa”).

\section{Discusión y conclusiones}

En este artículo, demostramos que los recolectores informales de desechos en Chile pueden otorgarle un sentido positivo a su trabajo, a pesar de la falta de protección, los bajos ingresos y la estigmatización que caracterizan esta actividad. Si bien es posible identificar al menos cinco experiencias-tipo, donde la precariedad es más o menos radical, en todas los recolectores parecen capaces de construir una imagen positiva de si mismos.

Siguiendo a Honneth, planteamos que la relación positiva de los recolectores con su trabajo se explica, en parte, por la aceptación de una "oferta ideológica de reconocimiento social", encarnada en la figura del "emprendedor". Desde las reformas neoliberales de los años 1980, las políticas públicas en Chile se han encargado de difundir esta oferta. Los gobiernos han erigido el "emprendimiento" como el ideal de contribución a la sociedad y forma privilegiada de superación de la pobreza. Esta retórica ha exaltado el rol individual en la realización social, disminuyendo la importancia del Estado en brindar las garantías materiales necesarias para este proceso, e instalando la lógica de la competencia como forma de regulación 
social. La reciente Ley de Fomento al Reciclaje encarna estos ideales. Los recolectores parecen haber aceptado su propuesta. Como sugiere Honneth, al identificarse con esta figura, los recolectores se sienten reconocidos simbólicamente por la sociedad, siendo innecesario e incluso contraproducente para esta identificación, el exigir que la estima tenga un correlato objetivo.

El artículo sugiere que la aceptación del discurso del emprendimiento por parte de los recolectores estaría condicionada por las limitaciones propias de su posición en el espacio social. Debido a su bajo capital cultural, social y económico, los recolectores experimentan en carne propia la clausura del sistema a lo largo de su trayectoria. A fuerza de fracasos y maltratos, aprenden a aceptar que el acceso a otra forma de estima social resulta improbable y abandonan sus expectativas de una integración social más sustancial. Si bien la organización de estos trabajadores podría permitirles eventualmente romper con este ciclo, esta alternativa sigue siendo por el momento menos atractiva que la identificación con la figura del emprendedor.

Estos hallazgos pueden servir para explicar por qué buena parte de los sectores más vulnerables en Chile no se han movilizado de manera abierta en contra las políticas neoliberales. El modelo neoliberal no sólo cumple con las expectativas de las clases medias al integrar sus demandas de autenticidad, como sugieren Boltanski y Chiapello (2010). También ha sido capaz de ofrecer a los trabajadores más pobres herramientas simbólicas para construir una identidad positiva de sí mismos, negándoles al mismo tiempo una integración social real. ¿En qué medida esta situación describe la realidad de otros países de la región? ¿Cuánto puede resistir en el tiempo una forma de realización que depende de un reconocimiento netamente simbólico? ¿Qué formas de reconocimiento ofrecen otros proyectos políticos? Estas preguntas abren varias líneas para investigaciones futuras.

\section{Referencias}

Aguilera, J., Salazar, R., Sepúlveda, M., Sepúlveda, M., \& Santelices, I. (2006). Residuos sólidos domiciliarios: logística, una herramienta moderna para enfrentar este antiguo problema. Revista Ingeniería Industrial, 5(1), 77-87.

Amuedo-Dorantes, C. (2004). Derterminants and Poverty Implications of Informal Sector Work in Chile. Economic Development and Cultural Change, 52(2), 347-368. https://doi.org/10.1086/380926

Asim. M, Batool, S., \& Chaudhry, M. (2012). Scavengers and their role in the recycling of waste in Southwestern Lahore. Resources, Conservation and Recycling, 58, 152-162. https://doi.org/10.1016/j.resconrec.2011.10.013

Becker, H. (1985). Outsiders. Etudes de sociologie de la déviance. París, Francia: Métailié.

Besiou, M., Georgiadis, P., \& Van Wassenhove, L. (2012). Official recycling and scavengers: Symbiotic or conflicting. European Journal of Operational Research, 218(2), 563-576. https://doi.org/10.1016/j.ejor.2011.11.030

Binion, E. \& Gutberlet, J. (2012). The effects of handling solid waste on the wellbeing of informal and organized recyclers: A review of the literature. International Journal of Occupational and Environmental Health 18(1), 43-52. https://doi.org/10.1179/1077352512Z.0000000001

Blau, D. (1985). Self-Employment and Self-Selection in Developing Country Labor Markets. Southern Economic Journal, 52(2), 351-363. https://doi.org/10.2307/1059621

Boltanski, L. \& Chiapello, E. (1999). Le nowvel espirit du capitalisme. París, Francia: Gallimard. Bräutigam, K., Gonzalez, T., Szanto, M., Seifert, H., \& y Vogdt, J. (2012). Municipal solid waste management in Santiago de Chile: challenges and perspectives towards sustainability. En: D. Heinrichs, K. Krellenberg, B. Hansjürgens, \& F. Martinez (Eds.), Risk Habitat Megacity (pp. 279-301). Heidelberg, Alemania: Springer. Carenzo, S. \& Fernández, M. (2011). El asociativismo como ejercicio de gubernamentalidad: "cartoneros/as" en la metrópolis de Buenos Aires. Argumentos, 24(65), 171-193. 
Francisca Gutiérrez, Stéphanie Alenda, Nicolás Angelcos, Satisfacción y reconocimiento en ocupaciones precarias: el caso de los recolectores de basura en Chile, Izquierdas, 49, junio 2020: 848-865

Carenzo, S. \& Míguez, P. (2010). De la atomización al asociativismo: Reflexiones en torno a los sentidos de la autogestión en experiencias asociativas desarrolladas por cartoneros. Maguaré, 24, 233-263.

Comisión Nacional del Medio Ambiente. (2010). Primer Reporte de Manejo de Residuos Sólidos en Chile. Santiago de Chile: CONAMA.

Cosacov, N. \& Perelman, M. (2015). Struggles over the Use of Public Space: Exploring Moralities and Narratives of Inequality. Cartoneros and Vecinos in Buenos Aires. Journal of Latin American Studies, 47(3), 521-542. https://doi.org/10.1017/S0022216X15000425

Do Carmo, M. \& Puppim de Oliveira, J. A. (2010). The Semantics of Garbage and the organization of the recyclers: Implementation challenges for establishing recycling cooperatives in the city of Rio de Janeiro, Brazil. Resources, Conservation and Recycling, 54(12), 1261-1268.

https://doi.org/10.1016/j.resconrec.2010.04.005

Fadda, G. \& Oviedo, E. (1994). Gestión local de servicios públicos en Chile: aseo, vivienda y educación. Los casos de Talca y Temuco. En: A. Rodríguez \& F. Velázquez (Eds.), Municipio y servicios públicos: Gobiernos locales en ciudades intermedias de América Latina (pp. 165-187). Santiago de Chile: Ediciones Sur. Ffrench-Davis, R. (2001). Entre el neoliberalismo y el crecimiento con equidad: tres décadas de política económica en Chile. Santiago de Chile: LOM Ediciones.

Garretón, M. A. (2012). Neoliberalismo corregido y progresismo limitado: los gobiernos de la concertación en Chile, 1990-2010. Santiago de Chile: Editorial Arcis/CLACSO.

Góngora, J. (2003). Beneficios y costos de políticas públicas ambientales en la gestión de residuos sólidos: Chile y Países seleccionados. Santiago de Chile: CEPAL.

Gutberlet, J. (2008). Empowering collective recycling initiatives: Video documentation and action research with a recycling co-op in Brazil. Resources, Conservation and Recycling, 52(4), 659-670. https://doi.org/10.1016/j.resconrec.2007.08.006

Gutberlet, J. \& Baeder, A. (2008). Informal Recycling and Occupational Health in Santo André, Brazil.

International Journal of Environmental Health Research, 18(1), 1-15. https://doi.org/10.1080/09603120701844258

Honneth, A. (2008). La société du mépris. Vers une nouvelle théorie critique. París, Francia: La découverte.

Honneth, A. (2010). La lutte pour la reconnaissance. París, Francia: Cerf.

Katusiimeh, M., Burger, K. \& Mol, A. (2013). Informal waste collection and its coexistence with the formal waste sector: The case of Kampala, Uganda. Habitat International, 38, 1-9. https://doi.org/10.1016/j.habitatint.2012.09.002

Magni, A. \& Günter, W. (2014). Cooperatives of Waste pickers as an alternative to social exclusion an its relationship with the homeless population. Saúde e Sociedade, 23(1), 99-109. http://dx.doi.org/10.1590/S0104-12902014000100011

Maloney, W. (2004). Informality Revisited. World Development, 32(7), 1159-1178. https://doi.org/10.1016/j.worlddev.2004.01.008

Medina, M. (1997). Informal Recycling and Collection of Solid Wastes in Developing Countries: Issues and Opportunities. Working Paper 24. Tokio: UNU/IAS.

Medina, M. (2000). Scavenger cooperatives in Asia and Latin America. Resources, Conservation and Recycling, 31(1), 51-69. https://doi.org/10.1016/S0921-3449(00)00071-9

Medina, M. (2001). Scavenging in America: back to the future? Resources, Conservation and Recycling, 31 (3), 229-240. https://doi.org/10.1016/S0921-3449(00)00082-3

Mesa para la Inclusión de los Recicladores de Base (2013). Políticas para la inclusión de los recicladores de base al sistema de gestión de residuos municipales en Chile. Santiago de Chile. https://www.ilo.org/wcmsp5/groups/public/---americas/---ro-lima/---sro-

santiago/documents/genericdocument/wcms_205403.pdf

Mitchell, C. (2008). Altered landscapes, altered livelihoods: The shifting experience of informal waste collecting during Hanoi's urban transition. Geoforum, 39(6), 2019-2029. https://doi.org/10.1016/j.geoforum.2008.07.006

Moulian, T. 2002. Chile actual. Anatomía de un mito. Santiago de Chile: LOM. 
Francisca Gutiérrez, Stéphanie Alenda, Nicolás Angelcos, Satisfacción y reconocimiento en ocupaciones precarias: el caso de los recolectores de basura en Chile, Izquierdas, 49, junio 2020: 848-865

Nas, P. \& Rivke, J. (2004). Informal Waste Management. Environment, Development and Sustainability, 6(3), 337-353.

https://doi.org/10.1023/B:ENVI.0000029912.41481.a5

Nzeadibe, C. \& Iwuoha, H. (2008). Informal waste recycling in Lagos, Nigeria. Communications in Waste \& Resources Management, 9(1), 24-30.

Nzeadibe, T. \& Ajaero, J. (2011). Development Impact of Advocacy initiatives in Solid Waste Management in Nigeria. Environment, Development and Sustainability, 13(1), 163-177.

https://doi.org/10.1007/s10668-010-9254-8

Packard, T. (2007). Do Workers in Chile Choose Informal Employment? A Dynamic Analysis of Sector Choice. Policy Research Working Paper 4232. Washington D.C: World Bank.

Paecke, S. (2004). Santiago: La difícil sustentabilidad de una ciudad neoliberal. En: C. De Mattos, M. Ducci, A. Rodríguez, \& G. Yáñez Warner (Eds.), Santiago en la globalización: ¿Una nueva Ciudad? (pp. 189215). Santiago de Chile: Ediciones Sur.

Paugam, S. (2009). La disqualification sociale. Essai sur la nouvelle pauvreté. París, Francia: PUF.

Perry, G., Maloney, W., Arias, O., Fajnzylber, P., Mason, A., Saavedra-Chanduvi, J., \& Bosch, M. (2007). Informalidad: escape y exclusión. World Bank Latin American and Carribean studies. Washington D.C: World Bank.

Pla, J. L. (2017). Trayectorias de clase y percepciones temporales sobre la posición ocupada en la estructura social. Un abordaje multidimensional de las clases sociales. Argentina 2003-2011. Revista internacional de sociología, 75(3). http://dx.doi.org/10.3989/ris.2017.75.3.16.05

Portes, A. \& Sassen-Koob, A. (1987). Making it Underground: Comparative Material on the Informal Sector in Western Market Economies. American Journal of Sociology, 93(1), 30-61. https://doi.org/10.1086/228705

Programa de las Naciones Unidas para el Desarrollo. (2017). Desiguales. Origenes, cambios y desafíos de la brecha social en Chile. Santiago de Chile: PNUD.

Rodríguez, A \& Rodríguez, P. (2013). Políticas neoliberales en Santiago de Chile: políticas contra la ciudad. Questiones urbano regionales. Revista del instituto de la Ciudad, 2(2), 9-31. http://hdl.handle.net/10469/6455

Sainz, J. (1998). The New Faces of Informality in Central America. Journal of Latin American Studies, 30(1), 157-179. https://doi.org/10.1017/S0022216X97004938

Salazar-Xirinachs, J. \& Chacaltana, J. (2018). Politicas de formalización en América Latina. Avances y desafíos. Lima: OIT.

Sánchez, G. (2018). Los efectos del afecto: un análisis posfundacional del discurso del emprendimiento de la Corfo. Tesis para optar al grado de Magíster en Sociología de la modernización, Facultad de Ciencias Sociales, Universidad de Chile.

Sembiring, E. \& Nitivattananon, V. (2010). Sustainable solid waste management toward an inclusive society: Integration of the informal sector. Resources, Conservation and Regycling, 54(11), 802-809. https://doi.org/10.1016/j.resconrec.2009.12.010

Wilson, D., Velis, C. \& Cheeseman, C. (2006). Role of informal sector recycling in waste management in developing countries. Habitat international, 30(4), 797-808.

https://doi.org/10.1016/j.habitatint.2005.09.005

Wilson, D., Adebisi, O., Chinwah, K., \& Cheeseman, C. (2009). Building recycling rates through the informal sector. Waste Management, 29(2), 629-635. https://doi.org/10.1016/j.wasman.2008.06.016 